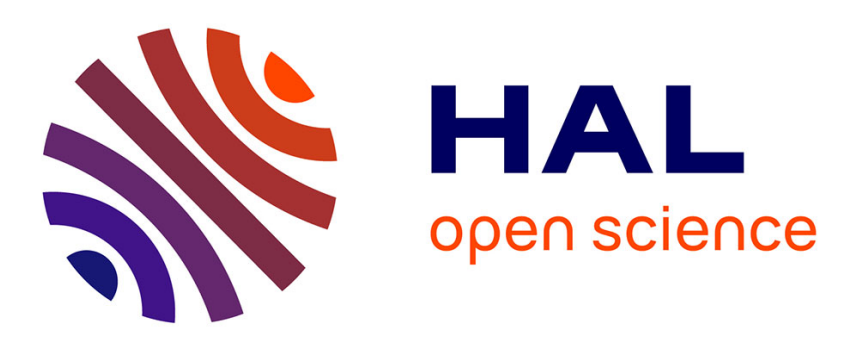

\title{
Les phénomènes de resyllabation chez les enfants en âge scolaire : perception d'erreurs et reformulation
}

Samantha Ruvoletto

\section{To cite this version:}

Samantha Ruvoletto. Les phénomènes de resyllabation chez les enfants en âge scolaire: perception d'erreurs et reformulation. Travaux de Linguistique: Revue Internationale de Linguistique Française, 2019, 10.3917/tl.079.0137 . hal-02884598

\section{HAL Id: hal-02884598 \\ https://hal.science/hal-02884598}

Submitted on 30 Jun 2020

HAL is a multi-disciplinary open access archive for the deposit and dissemination of scientific research documents, whether they are published or not. The documents may come from teaching and research institutions in France or abroad, or from public or private research centers.
L'archive ouverte pluridisciplinaire HAL, est destinée au dépôt et à la diffusion de documents scientifiques de niveau recherche, publiés ou non, émanant des établissements d'enseignement et de recherche français ou étrangers, des laboratoires publics ou privés. 


\title{
Les phénomènes de resyllabation chez les enfants en âge scolaire : perception d'erreurs et reformulation
}

\author{
SAMANTHA RUVOLETTO*
}

\begin{abstract}
Rédaction:
relu + biblio vérifiée, DONE

à faire: questions à l'auteure
\end{abstract}

\section{INTRODUCTION}

La liaison, l'élision et l'enchaînement sont des phénomènes linguistiques de resyllabation typiques de la langue française qui agissent sur une séquence de deux mots: Mot1, le mot de gauche et Mot2, le mot de droite. Dans les trois cas, le Mot2 est un mot à voyelle initiale, dont l'attaque syllabique est phonologiquement vide. Une consonne qui appartient au Mot1 va remplir l'attaque vide du Mot2 en provoquant la resyllabation qui implique un décalage entre frontières lexicales et frontières syllabiques de ces deux mots. La manière dont a lieu ce remplissage et la nature des segments impliqués permettent de distinguer ces trois phénomènes.

En considérant la définition de Schane (1967: 37) «l'élision est définie comme la suppression ou effacement de la voyelle finale d'un mot devant un autre mot commençant aussi par un son vocalique ». Pour montrer ce phénomène, Schane donne deux exemples : le $+a m i \rightarrow$ [lami] où l'élision a lieu et le + camarade $\rightarrow$ [le.ka.ma.кad] où le processus d'élision n'est pas enregistré. Comme exemplifié en [1], la voyelle finale du mot de gauche (Mot1) s'amuït devant un deuxième mot (Mot2) débutant par une voyelle. Ensuite la consonne qui était l'attaque du même mot devient l'attaque du Mot2, en conséquence de la chute d'un noyau vocalique.

[1] Mot $1:$ le [lo] + Mot $2:$ ours [uss] $\rightarrow$ l'ours [luss] où [ə] $\rightarrow \varnothing /$ V

L'enchaînement est un phénomène de resyllabation qui agit entre les frontières des mots qui constituent une seule unité prosodique. Dans le processus d'enchaînement, la consonne du Mot1 que nous pouvons appeler consonne fixe (CF), en utilisant la mention d'Encrevé (1983), va se lier

\footnotetext{
*Université de Lorraine; Campus Lettres et Sciences Humaines, 23 Boulevard Albert ${ }^{\text {er }}, 54000$ Nancy, France; samantha.ruvoletto@univ-lorraine.fr.
} 
par enchaînement à la voyelle initiale du Mot2 au moyen d'une resyllabation. Ainsi, la consonne qui est en position de coda dans la dernière syllabe du Mot1, se retrouve dans la position d'attaque de la syllabe suivante comme il est possible de voir en [2], où le deuxième [t] de petite [pə.tit] est la $\mathrm{CF}$.

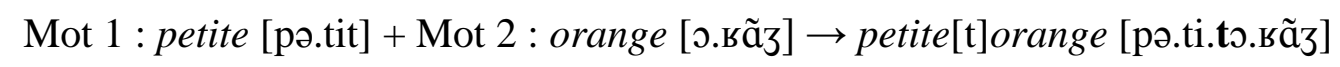

Dans cette opération, la CF ne change pas sa nature. Dans la quasi-totalité des cas d'enchaînement, il n'y a pas de modification de la mélodie du segment à quelques exceptions près (le [f] dans le couple neuf[v]ans qui est réalisé [v]; le [s] dans six[z]enfants qui se réalise en [z]). De plus dans l'enchaînement, la consonne du Motl qui s'enchaîne est toujours prononcée, avec ou sans enchaînement (la petite fille [la.pə.tit.fij]). Cette CF appartient aussi à la forme orthographique du Mot1 (petite [pə.tit]).

Contrairement à l'enchaînement impliquant une CF, dans le processus de liaison exemplifié en [3], une consonne phonologiquement latente (Encrevé, 1983) qui n'est pas toujours prononcée à l'oral, appelée consonne de liaison (CL) est phonétiquement réalisée (le [z] dans [le.za.mi] vs. les [le] en isolation où le $[\mathrm{z}]$ n'est pas acoustiquement réalisé).

[3] Mot1 : les [le] + Mot2:ami [ami] $\rightarrow$ les[z]ami [le.za.mi]

La CL est resyllabifiée dans le Mot2 si le mot commence par une voyelle (ex. les[z]amis [le.za.mi] vs. les garçons [le.gab.sõ]). La liaison donne également lieu à des changements au niveau segmental. Le premier changement phonétique est le dévoisement ou le voisement de la consonne en liaison. Par exemple, la CL fricative /s/ se voise et se réalise en [z] (ex. gros homme [gro.zom]) ainsi que l'occlusive /d/ se dévoise et devient [t] (ex. grand homme [gRã.tom]). De cette façon, même si, comme pour la CF, la CL est présente à l'orthographe à la fin de Mot2, sa réalisation ne correspond pas toujours au graphème qui la représente à l'écrit (le $<\mathrm{s}>$ de les est réalisé comme [z]). Le deuxième changement phonétique est lié à la réalisation des voyelles nasales. Nous avons deux réalisations possibles : une réalisation qui implique la dénasalisation de la voyelle nasale (ex. bon [bõ] réalisé [bon] dans bon ami [bo.na.mi]) ; une réalisation qui est le résultat d'une conservation de la nasalité sur la voyelle (ex. mon enfant [mõ.nã.fã]).

Dans l'exemple en [3], la CL est resyllabifiée dans Mot2. C'est le cas de la liaison dite « enchaînée » car une resyllabation est prévue. Mais, en français, nous pouvons aussi trouver des exemples de liaison «non-enchaînée ». Dans ce type de liaison, la resyllabation n'apparaît pas et la CL reste en position de coda du Mot1 (ex. vos amis [voz.a.mi]). Dans ce dernier cas, il y a une 
correspondance entre les frontières syllabiques et les frontières lexicales des mots car la CL garde la place qu'elle occupe dans la forme orthographique, soit la consonne finale du Mot1.

Plusieurs classifications de la liaison ont été proposées pour décrire sa variabilité. Les analyses du corpus Phonologie du Français Contemporain, abrégé PFC (Durand et Lyche, 2008) montrent que la liaison est presque toujours réalisée dans les contextes suivants : déterminant + nom (les[z]amis) ; proclitique + verbe (vous[z]allez), verbe + enclitique (allez-[z]y) ; expressions figées (de temps[z]en temps). En revanche, la liaison est très variable dans le contexte monosyllabique $+X$. Dans ce contexte, les pourcentages de réalisation changent selon le type de mots monosyllabiques : la liaison est presque toujours réalisée avec en (13 non-réalisations pour 2006 réalisations), beaucoup moins réalisée après dans (59 non-réalisations pour 859 réalisations) et encore plus variable pour chez (18 non-réalisations pour 74 réalisations). À la lumière des résultats du PFC, une classification de la liaison basée sur l'usage oral a été proposée (Durand et al., 2011). Cette classification prévoit deux types de liaison : une liaison catégorique, presque toujours réalisée par les locuteurs ; une liaison variable qui est sujet à une variation de réalisations selon différentes composantes (sociolinguistique, socioculturelle mais également statistique).

La description des phénomènes de resyllabation requiert aussi le traitement de cas plus spécifiques tels que le $h$ aspiré et le $h$ muet. Dans la forme graphique de certains mots, nous pouvons trouver un $h$ graphique qui est orthographiquement l'initiale du mot (ex. hippopotame, hibou, hasard). Cependant, ce $h$ orthographique n'a pas de réalisation phonétique correspondante (ex. hippopotame [i.po.po.tam]). Même si dans toutes les réalisations à l'oral de mots isolés commençant par $h$ nous n'avons aucune trace de cet élément, tous les mots qui commencent par $h$, ne sont pas identiques. Il y a des mots où le $h$ appartient seulement à la forme orthographique. Ces mots sont appelés « à $h$ muet initial » : le $h$ muet graphique est un indice de l'étymologie latine. Par exemple, dans le mot à $h$ muet initial honneur [о.пœе], le $h$ orthographique fait référence à la forme étymologique du mot latin honor. Dans ce cas, la liaison a bien lieu (les[z]hippopotame [le.zi.po.po.tam]). En revanche, il y d'autres mots où le $h$ initial n'est pas seulement orthographique mais implique des conséquences phonologiques. C'est le cas des mots dit à « $h$ aspiré initial ».

Pour définir le phénomène du $h$ aspiré initial, nous nous référons à l'article de Côté (2008 : 65).

«The so-called $h$ aspiré corresponds to the property of some morpheme-initial vowels to block processes that apply with normal initial vowels, and trigger processes that do not apply with regular vowels ».

En effet, la présence d'un Mot2 qui commence par un $h$ aspiré bloque la réalisation de la liaison : la consonne de liaison n'est pas réalisée [4a], contrairement à ce qui se passe devant voyelle [4b] : 
[4] a. $h$ aspiré : le(s) hérissons $\rightarrow$ [le.е.вi.sõ]

b. voyelle : les[z]abeilles $\rightarrow$ [le.za.brj]

La resyllabification qui a lieu dans ces trois phénomènes est responsable d'une noncorrespondance entre unités syllabiques et unités lexicales et d'un masquage des frontières des mots à l'oral qui rend difficile le processus de segmentation de la chaîne parlée chez les enfants francophones natifs pendant l'acquisition de leur langue maternelle. Plusieurs études (Chevrot et al., 2009 ; Dugua, 2006 ; Dugua et al., 2006 ; Nardy, 2008; Wauquier-Gravelines, 1996 ; WauquierGravelines, 2004 ; Wauquier, 2010) ont montré l'apparition d'erreurs dans les productions orales entre 2 et 3 ans qui reflètent une difficulté à déterminer la frontière gauche du Mot2, comme le remplacement de la CL par une consonne erronée (ex. de(s)[n]éléphants [de.ne.le.fã] pour des[z]éléphants [de.ze.le.fã]) ou, dans une moindre mesure, l'omission ou la non-réalisation de la

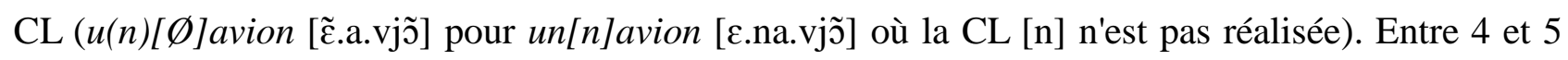
ans ces erreurs diminuent, avant de disparaître à l'âge de 6 ans comme montré par Basset (2000) et Dugua et Baclesse (2014) qui nous suggèrent qu'au-delà de l'âge de 7 ans, les enfants réalisent correctement plus de $97 \%$ des liaisons catégoriques.

Deux explications ont été proposées pour rendre compte de ce pattern développemental : une explication lexicale-constructioniste (Chevrot et al., 2007) appelée aussi Théorie Usage et Construction, TUC (Tomasello, 2003) et une explication phonologique autosegmentale (WauquierGravelines et Braud, 2005 ; Wauquier, 2009). Les deux explications sont en accord sur la description des premiers stades de l'acquisition de la liaison. En général, les enfants analysent d'abord les séquences en liaison présentes dans l'input comme une seule unité ([lezuss]). Ensuite ils commencent le découpage et ils interprètent la CL comme initiale du nom, sans établir une différence entre la CL et une consonne lexicale (la séquence [lezurs] est découpée comme [le] + [zurs] et le [z] n'a pas le statut particulier de la CL). À cause de cette analyse, les enfants commencent à produire les erreurs de liaison de remplacement que nous avons expliquées en détail dans le paragraphe précédent.

Les deux modèles donnent des interprétations différentes pour ce qui concerne le stade successif, quand la liaison vient d'être acquise. Pour le modèle TUC, acquérir des liaisons comme un[n]ours ou les[z]ours est accéder à des structures abstraites du type /un+nX/ ou /des+zX/, où -net -z-sont associés à la fin du Mot1 et au début du Mot2. De plus, il n'y a pas de différence entre la CL et la consonne lexicale, tout comme il n'y a pas de représentation sous-jacente spécifique pour la CL (Chevrot et al., 2013 ; Côté, 2005). Pour le modèle phonologique-autosegmentale, les enfants 
réussissent à abstraire et généraliser les séquences en liaison parce qu'ils acquièrent une représentation phonologique sous-jacente. Dans cette représentation (Figure 1), la CL fait surface au début du Mot2, car elle est associée à une position prosodique d'attaque mais, en même temps son contenu segmental est fourni par le Mot1, où il est stocké.

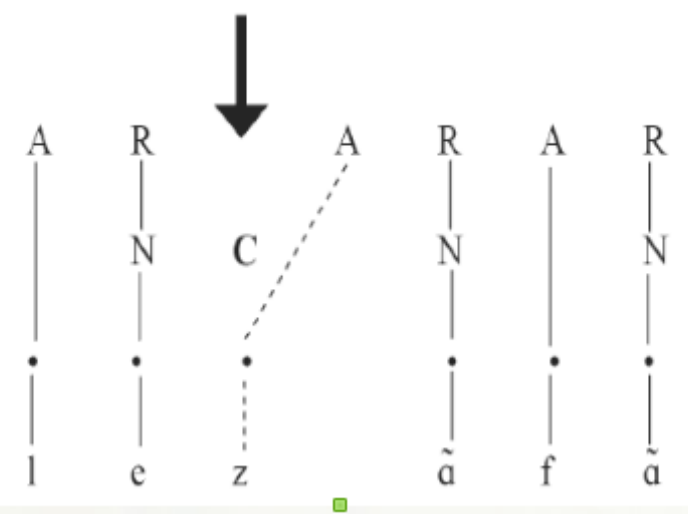

Figure 1. Schéma d'acquisition de la liaison: le stade final (Wauquier et Braud, 2005)

Mises à part les différentes modélisations de la dernière étape de l'acquisition de la liaison, pour les deux explications, la résolution des difficultés de réalisation des liaisons catégoriques est fixée entre 5 et 6 ans, âge du début de la scolarisation en France hexagonale.

Comme nous l'avons déjà dit précédemment, les études de Basset (2000) et de Dugua et Baclesse (2014) montrent qu'à partir de 6 ans les enfants ne réalisent plus d'erreurs lorsqu'ils doivent produire des liaisons catégoriques. Cependant, les données présentées dans ces recherches concernent exclusivement la liaison; elles ne permettent pas de comprendre si cette dynamique d'acquisition s'applique également aux autres phénomènes de resyllabation (comme l'élision ou l'enchaînement). De plus, il s'agit d'études qui analysent exclusivement l'aspect production. Pour la tranche d'âge de 5 à 7 ans, nous ne disposons pas de données longitudinales visant à comprendre la perception/compréhension ainsi que les erreurs, ni de résultats qui permettent de faire émerger les compétences métalinguistiques de ces enfants, mettant en rapport leurs connaissances linguistiques et l'éventuel «jugement» sur la réalisation de ces phénomènes. Pour ces raisons, nous avons décidé de recueillir ces données manquantes en proposant une tâche de perception d'erreurs et de reformulation utilisant des séquences de mots qui présentent des cas de liaison, d'élision et d'enchaînement.

Si la liaison ne pose plus de problèmes en production, nous visons à examiner dans notre étude si l'acquisition des phénomènes de resyllabation est véritablement terminée ou, en revanche, si les enfants qui commencent l'école primaire montrent encore des difficultés dans le traitement de 
mots en présence de liaisons catégoriques enchainées, d'élisions et d'enchaînements. Les données récoltées permettront d'enrichir le panorama sur l'acquisition de la liaison et de comprendre si son acquisition peut être généralisée à d'autres phénomènes impliquant une resyllabation.

\section{HYPOTHESES}

Pour vérifier si l'acquisition des phénomènes de resyllabation est complétée après 5 ans, nous proposons une tâche de perception d'erreurs et de reformulation. Cette tâche mettra en carte les éventuelles difficultés dans l'identification des erreurs de segmentation en cas de resyllabation et permettra ensuite d'observer également la production orale des séquences resyllabifiées en phase de reformulation.

Sur la base des résultats issus des études antérieures sur la production en cas de liaison, on peut s'attendre à ce que les enfants, à cet âge-là, n'aient plus aucune difficulté à identifier les erreurs de resyllabation et à les corriger. Nous nous attendions donc à ce qu'ils aient une bonne maîtrise à la fois de la liaison, de l'élision et de l'enchaînement et qu'ils n'éprouvent aucun problème dans la segmentation, aussi bien en perception qu'en production. Nous avons décidé d'élargir la portée de notre étude en poursuivant l'évolution des compétences pendant la première année de l'école primaire. À cet effet, nous avons comparé les résultats de deux groupes d'enfants, le premier se trouvant au début, le second à la fin du Cours Préparatoire $(\mathrm{CP})$, ce qui en France correspond à la première année de l'école primaire. Nous nous attendons à ce qu'à la fin du CP les erreurs de resyllabation soient plus facilement repérées et corrigées.

\section{PARTICIPANTS}

$\mathrm{Au}$ début de l'année scolaire, nous avons testé deux classes du CP (CP A et CP B) composées au total de 43 élèves (âge moyen : 6 ans et 3 mois, écart-type $=0,68$ ) d'une école primaire située dans le Nord de la France, à Dunkerque. Au moyen de questionnaires parentaux, nous avons récolté des données personnelles, linguistiques et socioéconomiques. Tous les enfants testés ont le français comme langue maternelle et sont issus de familles associées à des groupes socioculturels homogènes. Leurs connaissances de base concernant la langue orale et écrite, ainsi que d'autres compétences logique-cognitives ont été testées à l'entrée de l'école primaire par une évaluation globale proposée par le ministère de l’Éducation Nationale. L'analyse des résultats de 
cette évaluation a permis de vérifier que dans le groupe testé il n'y avait pas de participants ayant des difficultés ou des retards langagiers.

Au début du CP nous avons testé tous les enfants du groupe (43 enfants). À la fin du CP, pour des raisons de temps et de disponibilité des classes, nous avons testé seulement un échantillon de 12 enfants (âge moyen : 7 ans et 1 mois, écart-type =0,52) pour les deux classes ( 6 au CP A et 6 au CP B). Pour pouvoir disposer d'un échantillon représentatif, nous avons choisi les enfants à retester à la fin de l'année selon les résultats de la même tâche de perception d'erreurs et de reformulation au début du $\mathrm{CP}$, ce qui a permis la création de trois groupes de quatre enfants :

- Groupe $1(\mathrm{AM}, \mathrm{NB}, \mathrm{CMM}, \mathrm{CSW})^{1}: 4$ enfants qui ont eu des résultats au-dessus de la moyenne (2 dans le CP A et 2 dans le CP B) ;

- Groupe 2 (LT, ELF, CR, BW) : 4 enfants qui ont eu des résultats moyens ( 2 dans le CP A et 2 dans le CP B);

- Groupe 3 (EB, EM, COO, ERY) : 4 enfants qui ont eu des résultats au-dessous de la moyenne (2 dans le CP A et 2 dans le CP B).

\section{PROCÉDURE}

La tâche proposée mesure à la fois la perception d'une erreur «cible » insérée dans un énoncé à écouter et la correction que produit l'enfant sur sollicitation. D'abord, l'enfant, muni d'un casque audio, doit écouter une phrase prononcée par une locutrice de langue maternelle française. Après l'écoute de la phrase, l'enfant est prié de dire si la personne qui parle produit des fautes ou non. La consigne est la suivante : «on va écouter une dame qui ne parle pas trop bien français. Quelquefois elle fait des fautes. Toi, tu dois me dire si la phrase est juste ». Les réponses possibles sont « oui » ou «non ». Dans le premier cas, l'expérimentateur demande à l'enfant de corriger la faute repérée. La correction de la faute est très importante car la tâche que nous proposons est une tâche métalinguistique qui n'évalue pas seulement un type de compétence linguistique, phonologique en l'occurrence. Cette phase de correction permet, en effet, d'évaluer quels critères ont conduit au jugement qui a été émis. Les erreurs-cibles que nous avons insérées dans les phrases sont des erreurs phonologiques concernant les phénomènes de resyllabation (p. ex. le(s)[n]agneaux ou la armoire). Cependant, nous nous attendons aussi à ce que les enfants puissent considérer qu'une phrase est fautive pour des raisons sémantiques ou pragmatique plutôt que phonologiques. Par exemple, dans une phrase comme le artichaut est un bon légume (où l'erreur est dans la

1 Les sigles désignent les enfants de façon anonyme. 
production de le artichaut) l'enfant pourrait penser que la phrase est fautive parce qu'il considère tout simplement que les artichauts ne sont pas bons du tout. La correction des fautes nous guide dans le traitement des données et permet de comprendre si l'erreur-cible du phénomène de resyllabation est bien celle qui a conduit au rejet de la phrase.

Les enfants ont écouté les phrases au moyen d'un casque audio sennheiser hd 202 II au niveau de volume 16 sur 100. Les réponses ont été enregistrées par l'enregistreur Zoom H4 à une fréquence de $44000 \mathrm{~Hz}$ au format .wav. Les phrases ont été prononcées par une locutrice de langue maternelle française et enregistrées dans une chambre sourde à une fréquence de $44000 \mathrm{~Hz}$.

Les participants ont été testés un par un dans une salle silencieuse pendant 40 minutes. L'ordre des tâches et l'ordre de présentation du répertoire varient selon les enfants pour éviter des effets de facilitation ou de stratégies. Des pauses étaient prévues entre les différentes tâches et entre les blocs qui composent les tâches.

Des phases d'entraînement précédaient la passation proprement dite. Dans ces phases, l'expérimentateur expliquait à l'enfant la démarche et fournissait des exemples jusqu'à ce que la tâche soit comprise.

Entre début et fin de $\mathrm{CP}$, nous avons respecté le même ordre de présentation du matériel, la même procédure, ainsi que la façon de traiter les données.

\section{MATÉRIEL}

Nous avons proposé 48 phrases en français : 24 sont des phrases tests et 24 sont des fillers qui sont construits explicitement pour éviter que l'enfant comprenne le but de notre recherche. Les phrases tests et les phrases fillers sont mélangées en différentes séquences de présentation et regroupées en 6 blocs de 8 phrases. Nous résumons la séquence de présentation des phrases dans les différents blocs en annexe (Annexe 1). L'ordre de présentation des blocs de phrases a varié pour chaque enfant.

Les 24 phrases fillers (Annexe 2) sont des phrases grammaticales ou des phrases modifiées phonologiquement, c'est-à-dire par les processus de métathèse, d'épenthèse ou d'effacement de segments, ou syntaxiquement, par exemple par le non-respect de l'accord sujet-verbe.

Les phrases tests sont présentées dans 8 contextes phonologiques et syntaxiques. Les contextes syntaxiques choisis permettent la réalisation «catégorique » (cf. la terminologie introduite supra) et «fréquente » de phénomènes de resyllabation. Les contextes syntaxiques dans lesquels ont lieu les phénomènes de resyllabation sont au nombre de trois : déterminant-nom, verbe- 
enclitique et mot invariable-nom. L'élision et l'enchaînement sont toujours présentés dans le contexte syntaxique déterminant-nom. Les contextes phonologiques varient seulement pour la liaison qui est toujours une liaison enchainée. Pour cela nous avons créé quatre contextes phonologiques : liaisons en $[\mathrm{z}]$, en [n] et en [t], les CL le plus fréquents d'après l'analyse de Mallet (2008). Nous avons aussi des liaisons avec Mot2 qui commence par $h$ muet et $h$ aspiré. L'élision est toujours présentée dans le contexte phonologique $/ 1 /+\mathrm{V}$, alors que l'enchaînement se fait toujours dans un contexte en [n]. Tous les Mots $2^{2}$ choisis ont un Indice de Fréquence Standard (SFI) moyenne en MANULEX (Lété et al., 2004).

Les phénomènes de resyllabation dans les différents contextes phonologiques et syntaxiques sont présentés en trois conditions :

- Condition 1. Consonne erronée (CONDcons.err): Le(s)[n]agneaux [le.na.no] broutent l'herbe.

Entre les deux mots créant une resyllabation est insérée une consonne possible mais erronée (ex. [n] dans le(s)[n]agneaux [le.na.no] au lieu de [z] dans les[z]agneaux [le.za.no]). L'erreur-cible à identifier est une erreur d'insertion d'une consonne erronée.

Pour le cas de l'élision, nous n'avons pas pu faire cette insertion car le phénomène est d'une autre nature que la liaison et l'enchaînement. Pour cette raison, nous avons décidé de laisser dans la condition CONDcons.err un hiatus (ex. le artichaut).

— Condition 2. non-réalisation (CONDnon-réal) : Le(s)[Ø]anneaux [leØa.no] de cette chaîne sont cassés.

La phrase présente un contexte où le phénomène de resyllabation n'est pas réalisé $(L e(s)[\varnothing] a n n e a u x$ [leØa.no] au lieu de les[z]anneaux [le.za.no]). L'erreur à identifier est une erreur de non-réalisation du phénomène de resyllabation.

Pour l'élision, CONDnon-réal présente une phrase similaire à CONDcons.err, sauf que les voyelles en hiatus sont deux voyelles différentes ([a]-[a] pour CONDnon-réal : la armoire [lаØав.тwав] vs. [ə]-[а] pour CONDcons.err : le artichaut [ІәØав.ti.fo])

- Condition 3. Réalisation (CONDréal): Thibaut coupe des[z]ananas [de.za.na.na] en morceaux.

Le phénomène de resyllabation est réalisé. Aucune erreur ne doit être détectée.

\footnotetext{
${ }^{2}$ SFI correspond à un indice standardisé. Pour mieux comprendre cet indice, en voici un exemple. Si un mot a un SFI de 90, nous le rencontrons une seule fois tous les 10 mots lus. Si le mot a un SFI égal à 80, il est présent une fois tous les 100 mots lus.
} 
Dans le tableau 1, nous présentons le répertoire des phrases tests dans ces trois conditions. Pour presque tous les contextes, la phrase présentée dans la condition CONDréal est une phrase correcte tandis que dans les autres conditions elle présente des fautes. La seule exception est le contexte de liaison déterminant-nom avec $h$ aspiré pour CONDnon-réal et CONDréal. Ici, dans la condition CONDnon-réal, la phrase est correcte, car la liaison ne peut pas être réalisée. Par exemple, pour Le(s)[Ø]hamsters [leØam.stes] de mon cousin sont trop mignons, les enfants ne doivent pas repérer de fautes. En revanche, dans la condition CONDréal, la phrase comportant la réalisation de la liaison doit être considérée comme erronée, car en présence d'une liaison interdite. Par exemple, dans la phrase Paul et Marcel mangent des[z]hamburgers [de.zam.bюв.gœь], la séquence des[z]hamburgers [dе.zam.bœк.gœь] doit être signalée comme erronée.

Cette tâche est précédée d'un entraînement consistant en 10 phrases, qui sont présentées en Annexe 3. 


\begin{tabular}{|c|c|c|c|}
\hline Contextes & CONDcons.err & CONDnon-réal & CONDréal \\
\hline $\begin{array}{l}\text { Liaison en }[\mathrm{z}] \\
\text { déterminant-nom }\end{array}$ & $\begin{array}{c}\text { Le(s)[n]agneaux } \\
\text { [le.na.jo] broutent } \\
\text { l'herbe. }\end{array}$ & $\begin{array}{l}\text { Le(s)[Ø]anneaux } \\
\text { [leØa.no] de cette } \\
\text { chaîne sont cassés. }\end{array}$ & $\begin{array}{l}\text { Thibaut coupe } \\
\text { des[z]ananas } \\
\text { [de.za.na.na] en } \\
\text { morceaux. }\end{array}$ \\
\hline $\begin{array}{l}\text { Liaison en }[\mathrm{n}] \\
\text { déterminant-nom }\end{array}$ & 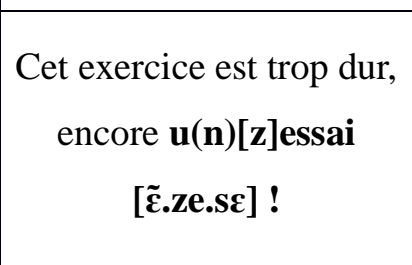 & 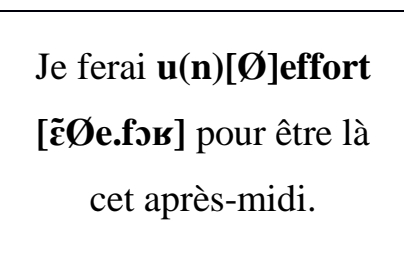 & 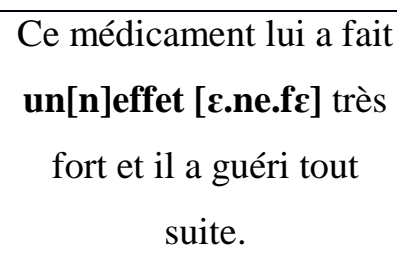 \\
\hline $\begin{array}{l}\text { Liaison verbe- } \\
\text { enclitique }\end{array}$ & $\begin{array}{c}\mathbf{E s}(\mathbf{t})-[\mathbf{z}] \mathbf{o n}[\varepsilon . z \tilde{\mathbf{z}}] \text { bientôt } \\
\text { arrivés à la maison ? }\end{array}$ & $\begin{array}{c}\mathbf{E s}(\mathbf{t})[\emptyset] \text {-on }[\varepsilon \emptyset \tilde{\mathbf{z}}] \text { déjà } \\
\text { arrivés ? }\end{array}$ & $\begin{array}{c}\text { Peut[t]-on [pø.t̃̃] savoir } \\
\text { tes secrets? }\end{array}$ \\
\hline $\begin{array}{l}\text { Liaison mot invariable } \\
\text {-nom }\end{array}$ & 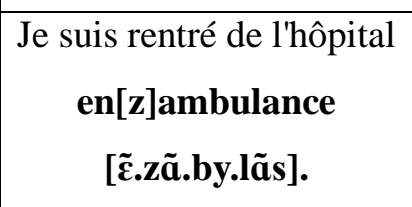 & 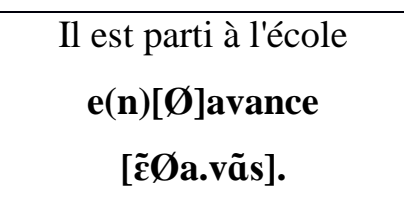 & $\begin{array}{c}\text { Ce livre est } \\
\text { très[z]ennuyeux } \\
\text { [tкع.zã.nui.jø]. }\end{array}$ \\
\hline $\begin{array}{l}\text { Liaison dét-nom avec } \\
h \text { muet }\end{array}$ & $\begin{array}{c}\text { Ma sœur doit s'acheter } \\
\operatorname{de}(\mathbf{s})[\text { n]habits } \\
\text { [de.na.bi]. }\end{array}$ & $\begin{array}{c}\text { Le(s)[Ø]habitudes } \\
\text { [leØa.bi.tyd] de ce } \\
\text { chien doivent changer, il } \\
\text { n'est pas sage! }\end{array}$ & $\begin{array}{c}\text { Les[z]habitants } \\
\text { [le.za.bi.tã] de Paris } \\
\text { s'appellent les Parisiens. }\end{array}$ \\
\hline $\begin{array}{l}\text { Liaison dét-nom avec } \\
h \text { aspiré }\end{array}$ & $\begin{array}{c}\text { On a trouvé } \\
\operatorname{de}(\mathbf{s})[\mathbf{n}] \text { haricots } \\
\text { [de.na.ki.ko] dans le } \\
\text { jardin. }\end{array}$ & $\begin{array}{l}\text { Le(s)[Ø]hamsters } \\
\text { [leØam.ster] de mon } \\
\text { cousin sont trop } \\
\text { mignons. }\end{array}$ & $\begin{array}{c}\text { Paul et Marcel mangent } \\
\text { des[z]hamburgers } \\
\text { [de.zam.ьœк.gœь]. }\end{array}$ \\
\hline $\begin{array}{l}\text { Enchaînement dét- } \\
\text { nom }\end{array}$ & $\begin{array}{c}\text { Paul a oublié } \\
\text { une[z]affaire } \\
\text { [yn.za.fers] à l'école. }\end{array}$ & $\begin{array}{l}\text { Un(e) agence } \\
\text { [ynØa.zãs] de voyage } \\
\text { est un magasin où ils } \\
\text { organisent des vacances }\end{array}$ & $\begin{array}{l}\text { Maxime a vu une } \\
\text { affiche [y.na.fij] du } \\
\text { cirque. }\end{array}$ \\
\hline Élision dét.-nom & $\begin{array}{c}\text { Le artichaut } \\
\text { [ІӘØак.ti.fo] est un bon } \\
\text { légume. }\end{array}$ & $\begin{array}{c}\text { La armoire } \\
\text { [laØак.mwar] de ma } \\
\text { chambre est bien rangée. }\end{array}$ & $\begin{array}{l}\text { J'ai découpé l'article } \\
\text { [las.tikl] sur les lions } \\
\text { dans mon journal. }\end{array}$ \\
\hline
\end{tabular}




\section{LE TRAITEMENT DE DONNÉES}

Pour la tâche de perception d'erreurs, si nous présentons une phrase juste ou une phrase fausse, nous avons deux réponses possibles qui donnent lieu à deux évaluations possibles (correcte ou erronée), comme le montre le Tableau 2 :

\begin{tabular}{|c|c|c|}
\hline Phrase & Réponse enfant & Évaluation donnée \\
\hline \multirow{2}{*}{ Juste } & Oui & Correcte \\
\cline { 2 - 3 } & Non & Erronée \\
\hline \multirow{2}{*}{ Fausse } & Non & Correcte \\
\cline { 2 - 3 } & Oui & Erronée \\
\hline
\end{tabular}

Tableau 2. Réponses possibles et évaluations données

Dans une première analyse 'brute' de nos résultats, les réponses «non » à des phrases fausses et «oui » à des phrases justes doivent être évaluées comme des réponses correctes. En revanche, les réponses «oui » à des phrases fausses et «non» à des phrases justes doivent être comptées comme des erreurs. Ce sont les résultats de ce type d'analyse que nous présentons d'abord.

Toutefois, la tâche ne se limite pas seulement à l'enregistrement des réponses « oui » et «non ». Elle se compose aussi d'une partie de reformulation qui permet de rendre compte des critères utilisés par l'enfant dans son jugement et de vérifier l'identification de l'erreur-cible. Nous pouvons distinguer des réponses basées sur des critères plutôt sémantiques (les enfants trouvent des erreurs sémantiques dans la phrase) ou lexicaux (les enfants considèrent comme fausse une phrase parce qu'elle contient un mot qu'ils ne connaissent pas). Les élèves peuvent aussi ne pas parvenir à motiver leur jugement. L'examen de la correction d'erreurs permet d'éliminer, dans le total brut, les fautes qui ne nous intéressent pas, comme par exemple les fautes sémantico-lexicales ou les fautes sans motivation, pour affiner l'analyse brute.

Nous proposons une analyse qualitative et quantitative des données au moyen de plusieurs tests statistiques. D’abord nous allons présenter les données de 43 enfants testés au début du CP. Comme nous l'avons annoncé dans la description des participants, à la fin du CP nous n'avons pas pu re-tester la totalité des 43 enfants, mais seulement un échantillon représentatif de 12 enfants issus du groupe de départ. Nous avons donc pu proposer dans cet article une comparaison des résultats de l'échantillon des 12 enfants en question, au début et à la fin du CP, qui permet de décrire leur évolution. 


\section{RÉSULTATS}

Comme nous l'avons dit dans le paragraphe précédent, les premiers résultats que nous présentons sont des résultats bruts qui ne tiennent pas compte des reformulations produites par les enfants. Nous avons calculé les scores moyens de réponses correctes pour chaque condition (Tableau 3). Le score va de 0 (0 réponses correctes) à 8 (toutes les réponses sont correctes). Nous avons écarté des résultats les données de deux enfants (4,6\%) qui ne comprenaient pas les instructions.

\begin{tabular}{|c|c|}
\hline Conditions & Scores moyens de réponses correctes \\
\hline CONDcons.err (le(s)[n]agneaux) & 3,7 \\
\hline CONDnon-réal (le(s)[Ø]anneaux) & 4,07 \\
\hline CONDréal (les[z]ananas) & 6,50 \\
\hline
\end{tabular}

Tableau n 3. Résultats de la tache au début du CP pour les trois conditions. Le score maximal est 8.

L'analyse de la variance (ANOVA) des scores de réponses correctes dans trois modalités (types de conditions) utilisant les sujets et les items comme variables aléatoires montre un effet du facteur « condition » $\left(\mathrm{F}_{1}(2,80)=21,08, \mathrm{p}<.05 ; \mathrm{F}_{2}(2,14)=7,490, \mathrm{p}<.001\right)$. L'analyse des contrastes montre que la CONDréal implique plus de réponses correctes que les deux autres conditions (CONDcons.err vs. CONDnon-réal $\mathrm{F}_{1}(1,40)=.32, \mathrm{p}=.57 ; \mathrm{F}_{2}(1,7)=.017, \mathrm{p}=.96 ;$ CONDcons.err et CONDnon-réal vs. CONDréal $\left.\mathrm{F}_{1}(1,40)=52,67 \mathrm{p}<.05 ; \mathrm{F}_{2}(1,7)=14,402, \mathrm{p}<.001\right)$.

Dans la condition CONDréal, les enfants doivent juger la phrase comme correcte. La seule exception est la condition « liaison déterminant-nom avec $h$ aspiré », où la réalisation de la liaison doit être considérée comme une erreur et la phrase correspondante doit être traitée comme fausse. En dehors du contexte particulier avec $h$ aspiré, la plupart des enfants qui trouvent une faute ne donnent aucune motivation ou trouvent des erreurs sémantiques ou lexicales. Par exemple, dans la

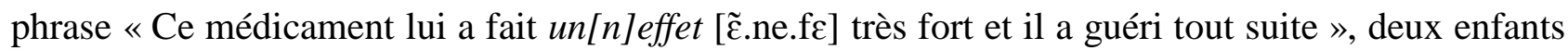
nous disent que la phrase est erronée parce que les médicaments ne font pas un effet tout de suite mais qu' « il faut attendre ». Pour la phrase avec élision «J'ai découpé l'article [lak.tikl] sur les lions dans mon journal », les quatre enfants qui signalent des erreurs nous suggèrent qu'on ne découpe

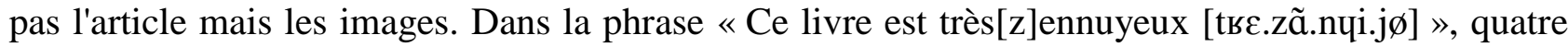
enfants ne considèrent pas la possibilité qu'un livre puisse être ennuyeux. Puisque les enfants ne repèrent pas de problèmes phonologiques dans cette condition, nous ne proposons pas une analyse 
détaillée, contrairement à ce que nous allons faire pour CONDcons.err ou CONDnon- réal dans la section suivante.

\subsection{La détection d'erreurs de non-réalisation et d'ajout d'une consonne erronée}

Pour affiner le traitement des données nous avons analysé les reformulations pour les classer en différents groupes :

— Groupe «sémantique»: dans ce groupe, nous considérons les enfants qui trouvent des erreurs sémantiques ou lexicales dans la phrase test.

- Groupe «sans motivation »: ce groupe comprend les enfants qui ne motivent pas la réponse : ils trouvent que la phrase n'est pas bonne, mais sont incapables de dire pourquoi.

— Groupe «cibles »: dans ce groupe, nous mettons les enfants qui ont détecté l'erreur « cible $»$.

Dans le tableau 4 nous présentons les résultats et la classification reprenant le nombre d'enfants pour chaque groupe pour les deux conditions.

\begin{tabular}{|c|c|c|c|c|c|c|c|}
\hline & \multicolumn{6}{|c|}{$\begin{array}{l}\text { Nombre d'enfants (max. 43) qui identifient une erreur du } \\
\text { type : }\end{array}$} \\
\hline & & \multicolumn{2}{|c|}{ sémantique } & \multicolumn{2}{|c|}{ sans motivation } & \multicolumn{2}{|c|}{ cible } \\
\hline & & $\begin{array}{l}\text { CONDno } \\
\text { n-réal }\end{array}$ & $\begin{array}{l}\text { CONDco } \\
\text { ns.err }\end{array}$ & $\begin{array}{c}\text { CONDno } \\
\text { n-réal }\end{array}$ & $\begin{array}{c}\text { CONDco } \\
\text { ns.err }\end{array}$ & $\begin{array}{c}\text { CONDno } \\
\text { n-réal }\end{array}$ & $\begin{array}{c}\text { CONDco } \\
\text { ns.err }\end{array}$ \\
\hline \multirow{6}{*}{ Liaison } & en $[z]$ & 1 & 5 & 2 & 2 & 5 & 18 \\
\hline & en $[\mathrm{n}]$ & 2 & 0 & 1 & 4 & 17 & 22 \\
\hline & verbe- enclitique & 11 & 7 & 2 & 2 & 9 & 9 \\
\hline & mot invariable-nom & 2 & 3 & 3 & 0 & 19 & 26 \\
\hline & avec $h$ muet & 3 & 3 & 2 & 2 & 12 & 24 \\
\hline & avec $h$ aspiré & 0 & 3 & 0 & 2 & 6 & 28 \\
\hline \multicolumn{2}{|c|}{ Enchaînement } & 6 & 0 & 6 & 2 & 6 & 32 \\
\hline \multicolumn{2}{|l|}{ Élision } & 6 & 3 & 4 & 2 & 13 & 12 \\
\hline
\end{tabular}

Tableau $n$ 4. Classification des erreurs des enfants après l'analyse des reformulations pour les deux conditions 
Dans la condition CONDcons.err, la plupart des enfants réussissent à détecter l'erreur-cible. Cependant, nous remarquons des enfants qui signalent des erreurs sémantiques. Trois enfants jugent la première phrase «Le(s)[n]agneaux [le.na.no] broutent l'herbe » comme fausse parce que ce ne sont pas les agneaux qui broutent l'herbe mais plutôt les moutons. L'enfant NB prétend que les agneaux ne broutent pas l'herbe mais qu'ils sentent plutôt l'herbe. Dans la phrase « Ma sœur doit s'acheter de(s)[n]habits [de.na.bi] », pour l'enfant EF, la faute n'est pas dans la CL erronée mais dans le fait qu'on n'achète pas des habits mais des vêtements. Enfin, pour donner un dernier exemple, l'enfant CDL nous apprend qu'on ne trouve pas des haricots dans le jardin mais des abricots et que c'est pour cela que, selon lui, la phrase « On a trouvés de(s)[n]haricots [de.na.si.ko] dans le jardin » est erronée.

Dans CONDnon-réal, les erreurs sémantiques et lexicales sont plus fréquentes que dans CONDcons.err. La plupart des erreurs sémantiques et lexicales sont enregistrées dans le contexte

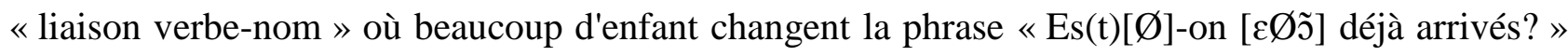
en proposant des réinterprétations comme «des gens est déjà arrivé ». Dans le contexte d'enchaînement déterminant-nom, l'agence de voyage devient pour un enfant « une agence du cinéma » ou pour un autre «une agence de voyage pour les vacances ». Pour la non-réalisation d'élision, la armoire bien rangée devient «pas rangée » ou «pas mangée ». En contexte de liaison déterminant-nom en $h$ aspiré, les enfants SV et LT, nous disent que la phrase est fausse car les habitudes d'un chien ne peuvent pas changer et l'enfant IV nous répond « il y a une faute parce que si le chien n'est pas sage, on donne des coups de martinet ».

Comme notre recherche concerne les phénomènes de resyllabation, nous voulons voir si dans cette tâche au CP les enfants sont capables de reconnaître les insertions abusives de consonnes et les non-réalisations. À cet effet, nous proposons ici les pourcentages d'«identification»de l'erreur-cible (Tableau 5) : 


\begin{tabular}{|c|c|c|c|}
\hline & & $\%$ d'identificati & le l'erreur-cible \\
\hline & & CONDcons.err & CONDnon-réal \\
\hline & Déterminant-nom (en [z]) & 41,8 & 34,9 \\
\hline & Déterminant-nom (en [n]) & 46,5 & 49,5 \\
\hline & Verbe-enclitique & 51,2 & 20,9 \\
\hline Llaisui & Mot invariable-nom & 55,8 & 41 \\
\hline & $h$ muet & 39,5 & 27,9 \\
\hline & $h$ aspiré & 13,5 & 13,9 \\
\hline Enchaîn & & 39,5 & 13,9 \\
\hline Élisions & & 53,4 & 30,2 \\
\hline
\end{tabular}

Tableau 5. Identifications de l'erreur-cible pour CONDcons.err et pour CONDnon-réal

Pour CONDcons.err, les contextes qui créent le plus de problèmes (moins de $50 \%$ d'identifications) correspondent aux phrases où on présente une erreur de liaison entre déterminant et nom (en [z] 41,8 \%, en [n] 46,5 \%, $h$ muet 39,5 \%) et une erreur d'enchaînement (39,5 \%). Pour les autres contextes, les enfants parviennent plus facilement à repérer l'erreur-cible. Nous remarquons que, dans le dernier contexte « liaison $h$ aspiré », 13,5\% des enfants reconnaissent la faute de CL erronée mais à la demande de reformulation, 60,7\% produisent une liaison avec $h$ aspiré du type le[z]haricots. La reformulation de la phrase dans ce cas-là correspond à une production erronée car la liaison avec $h$ aspiré n'est pas phonologiquement possible en français.

Pour comprendre le traitement dans la deuxième condition (CONDnon-réal), il faut d'abord faire une distinction entre le contexte « liaison entre déterminant-nom avec $h$ aspiré » et les autres contextes. Dans CONDnon-réal, la phrase avec Mot2 commençant par $h$ aspiré est correcte. Les enfants qui n'identifient pas de faute dans ce contexte (par exemple dans la phrase «Le(s)[Ø]hamsters [leØam.stes] de mon cousin sont trop mignons ») répondent correctement. Au contraire, les enfants qui trouvent une faute dans ce contexte ne répondent pas correctement, car la non-réalisation de liaison avec $h$ aspiré est une production correcte et la phrase doit être jugée comme juste. Les 6 enfants dans le tableau qui ont identifié une faute (soit 13,9\%) considèrent le contexte avec $h$ aspiré comme le contexte avec $h$ muet, en réalisant la liaison en [z] dans la phase de correction. Pour la condition non-réalisation, dans tous les contextes les enfants identifient très difficilement l'erreur-cible (moins de $50 \%$ d'identifications). Les enfants acceptent plus facilement 
la non-réalisation dans les contextes: liaison verbe-enclitique (20,9\% d'identifications), enchaînement déterminant-nom (13,9\%), élision déterminant-nom (30,2 \%) et liaison déterminantnom avec $h$ muet $(27,9 \%)$.

\subsection{Comparaison entre les deux conditions : CONDnon-réal et CONDcons.err}

Nous avons vu que les enfants ont des difficultés pour CONDnon-réal et CONDcons.err. Cela veut dire que, pour les enfants, certaines non-réalisations et certaines utilisations de consonnes erronées sont acceptables dans la langue orale. Nous allons comparer les cas où les enfants ont identifié des erreurs phonologiques (erreurs-cibles) pour la CONDcons.err et pour la CONDnonréal, afin de comprendre comment ils jugent ces deux façons de traiter les frontières de mot en fonction du phénomène de resyllabation testé.

Nous comparons les scores d'identification de l'erreur-cible sur les deux CONDnon-réal et CONDcons.err (le score maximal est de 8) et nous observons qu'il y a une différence statistiquement significative pour la liaison $\left(\mathrm{t}_{1}(40)=2,49, \mathrm{p}<.05, \mathrm{t}_{2}(5)=0,30, \mathrm{p}<.05\right)$ et pour l'enchaînement $\left(\mathrm{t}_{1}(40)=5,92 \mathrm{p}<.05^{3}\right)$ mais pas pour l'élision $\left(\mathrm{t}_{1}(40)=1,53, \mathrm{p}=.13\right)$. Le graphique 1 montre clairement que le score moyen d'identification de l'erreur-cible est plus élevé dans CONDcons.err pour ces deux phénomènes.

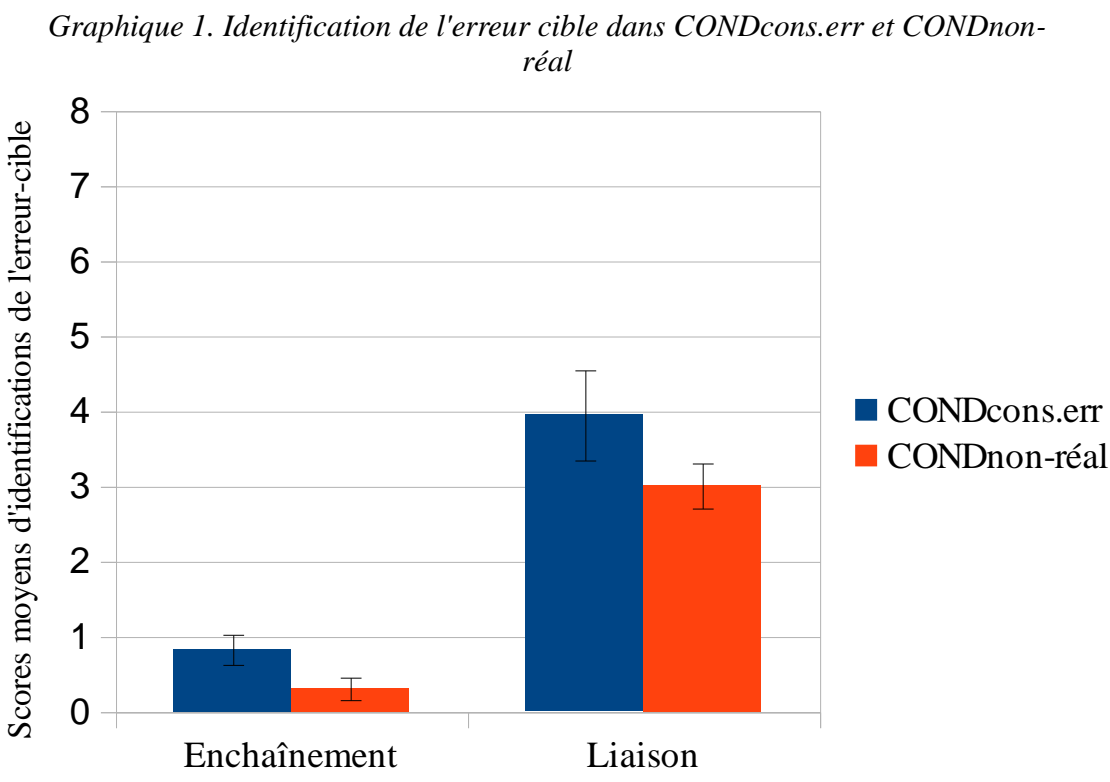

3 Pour l'élision et l'enchaînement, nous avons seulement un item par condition. Dès lors, nous ne pouvons pas calculer le $\mathrm{t}_{2}$. 
Cela veut dire qu'à l'oral, pour l'enchaînement et la liaison, les enfants au début du CP acceptent davantage une non-réalisation que l'ajout d'un segment erroné. Ils acceptent plus facilement une construction qui présente les mots dans leurs formes lexicales séparées et qui garde leurs frontières intactes ([leØa.no]) qu'une construction qui altère ces formes lexicales.

Pour l'élision, nous avons remarqué les mêmes difficultés pour les deux conditions. En effet, nous proposons dans les deux cas un jugement sur des séquences similaires, où nous ajoutons une pause entre déterminant et nom : soit le artichaut (CONDcons.err), soit la armoire (CONDnonréal). Ces deux constructions sont perçues de la même façon et jugées avec la même difficulté, car il n'y a pas une véritable différence dans le traitement des frontières entre mots. Ce résultat fait émerger une problématique liée à la construction de la tâche. Nous allons décrire plus en détail les limites de cette tâche dans la discussion. Étant donné les limites des items en élision, nous avons préféré discuter séparément les résultats liés à ce phénomène dans la section qui suit.

\subsection{L'évolution des résultats : début du CP et fin du CP}

Nous avons établi une comparaison des résultats pour l'échantillon de 12 enfants testés au début et à la fin du CP. Nous avons considéré les scores des réponses correctes (les identifications d'erreurs-cibles concernant l'ajout d'une consonne erronée dans CONDcons.err, la non-réalisation dans CONDnon-réal, ainsi que les non-identifications d'erreurs dans la condition de réalisation CONDréal) pour la liaison et l'enchaînement. Au moyen d'un t-test, nous pouvons établir une différence statistiquement significative et donc une amélioration significative entre les scores de la tâche au début et à la fin de l'année, mais seulement pour la condition CONDcons.err $\left(\mathrm{t}_{1}(12)=0,96\right.$, $\left.\mathrm{p}<.05, \mathrm{t}_{2}(6)=1,20, \mathrm{p}<.05\right)$. Cette amélioration n'est pas observée pour CONDréal $\left(\mathrm{t}_{1}(11)=1,86\right.$, $\left.\mathrm{p}=1,00, \mathrm{t}_{2}(6)=1,04, \mathrm{p}=.32\right)$, ni pour CONDnon-réal $\left(\mathrm{t}_{1}(11)=1,07, \mathrm{p}=0,30, \mathrm{t}_{2}(6)=0,80\right.$, $\mathrm{p}=.44$ ), comme cela est bien représenté dans le graphique 2 (le score maximal est 7). Cependant, ce résultat peut être dû à un 'effet plafond', les scores enregistrés pour le test effectué en CP pour les deux conditions étant déjà assez élevés. Nous pouvons donc penser que le score a déjà atteint son maximum de fait et qu'aucun changement ne pourrait encore conduire à une amélioration. 


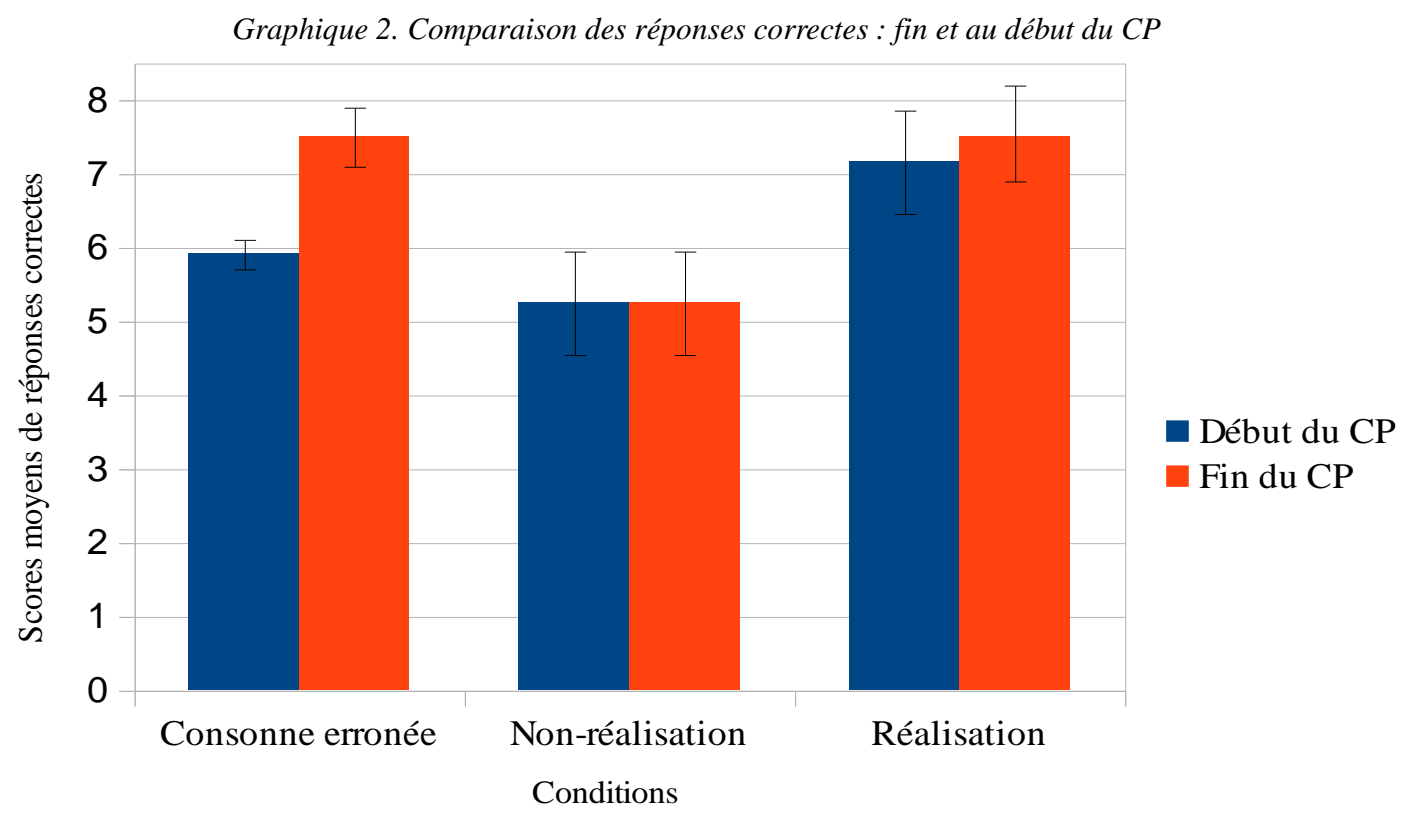

Les enfants à la fin du $\mathrm{CP}$ parviennent donc à mieux repérer l'insertion d'une consonne erronée entre Mot1 et Mot2. Cependant, au début et à la fin du CP, ils éprouvent les mêmes difficultés à signaler les non-réalisations et à considérer celles-ci comme des fautes.

\section{DISCUSSION ET CONCLUSIONS}

Comme nous l'avons présenté dans l'introduction, plusieurs études en acquisition montrent que les enfants français entre 5 et 6 ans traitent correctement la consonne de liaison (CL) et parviennent à la distinguer d'une consonne lexicale. Le traitement correct de la CL implique la résolution des erreurs de liaison en production, indépendamment du modèle théorique qu'on utilise pour expliquer l'acquisition de ces phénomènes.

Nous avons testé des enfants au début du CP (6 ans d'âge) au moyen d'une tâche de perception d'erreurs suivie de reformulation. Pendant la tâche, les enfants écoutent des phrases caractérisées pas des erreurs-cibles de resyllabation: l'ajout d'une consonne erronée (ex. $l e(s)[n]$ agneaux [le.na.no]) et la non-réalisation d'un phénomène de resyllabation (ex. les[Ø]anneaux [le.a.no]). Nous avons d'abord écarté les erreurs sémantiques et lexicales, pour ensuite nous focaliser sur l'identification des erreurs-cibles. Nous avons, conformément à la littérature en production pour la liaison, fait l'hypothèse qu'aucun problème de segmentation ne devait plus exister en perception sur les frontières resyllabées, non seulement en cas de liaison, mais également en cas d'élision ou dans les enchaînements. Pourtant, contrairement aux hypothèses, à la 
fin comme au début du $\mathrm{CP}$, nous avons encore constaté des difficultés à détecter les non-réalisations dans la tâche de perception d'erreurs pour les différents phénomènes. Au début et à la fin du $\mathrm{CP}$, il est encore très difficile pour les enfants de juger comme erronée une séquence où le phénomène de resyllabation n'est pas produit (pour la liaison les[Ø]ours [leØuss], pour l'enchaînement uneambulance [yn.ã.by.lãs]), indépendamment du contexte syntaxique. Néanmoins, les séquences de Mot1 et Mot2 caractérisées par l'insertion d'une consonne erronée en cas de liaison et d'enchaînement sont plus fréquemment repérées comme fautives, ce qui laisse à penser que les enfants savent qu'elles ne sont pas acceptables.

Nos résultats sur les non-réalisations remettent en question nos hypothèses basées sur les études précédentes portant sur l'acquisition de la liaison qui prennent en compte exclusivement les productions des enfants. Même si les enfants à 6 ans réussissent à produire correctement la resyllabation, nous remarquons qu'ils ont encore des difficultés à détecter et à juger correctement la réalisation de ces différents phénomènes. Ces résultats nous permettent de nous interroger sur la définition de l'acquisition des phénomènes étudiés. Pour déterminer si leur acquisition est complète, il n'est pas suffisant d'analyser leur production à un moment donné, mais il est préférable d'investiguer aussi leur compréhension et leur perception afin d'avoir un cadre complet permettant la création de modèles explicatifs.

Contrairement aux données en production, nos résultats soulignent l'importance dans le processus d'acquisition des non-réalisations des phénomènes de resyllabation. Il ne nous semble pas que l'acceptation des non-réalisations est une conséquence de l'input adulte entendu par les enfants lorsqu'ils apprennent leur langue maternelle car, comme on l'a vu dans l'introduction, la liaison est presque toujours réalisée dans les contextes testés. Étant donné qu'à l'âge testé les enfants commencent l'apprentissage des formes écrites des mots, nous pouvons prendre en compte cette variable pour expliquer nos résultats. Quand les enfants apprennent à écrire, les séquences entendues à l'oral prennent forme sur papier. À l'écrit, les frontières des mots sont bien délimitées par des symboles graphiques comme des espaces blancs (ex. forme orthographique <les ours>) ou des apostrophes (ex. <l'éléphant>). Nous pouvons penser que cet apprentissage aurait une influence négative sur les productions orales : apprendre Mot1 + Mot2 comme deux formes séparées à l'écrit (ex. <les ours>) impliquerait de les produire séparément, sans réaliser les phénomènes de resyllabation à l'oral, mais aussi d'accepter cette non-réalisation. L'apprentissage de l'orthographe chez les petits Français au début de l'école primaire peut donc être vu comme un facteur qui est en lien avec la stabilité de la non-réalisation en perception, mais il peut également être responsable des résultats meilleurs dans l'identification d'erreurs d'insertions de consonnes erronées. À l'écrit, la consonne qui resyllabifie sur Mot2 est orthographiée comme un graphème à la fin de Mot1 (par 
exemple, le $\langle\mathrm{s}>$ dans $\langle$ les ours $>$ ou le deuxième $\langle\mathrm{t}\rangle$ dans $<$ petite amie $>$ ). Associer le son à la lettre pendant l'apprentissage de la langue écrite permettrait de rendre encore plus évidentes et saillantes les erreurs d'insertion d'une consonne erronée. Cette idée de mettre en rapport la langue orale et l'apprentissage de la langue écrite n'est pas nouvelle car plusieurs études ont montré qu'apprendre la forme orthographique des mots influence la représentation phonologique qui y est associée (Chevrot, 1996 ; Peereman et al., 2009) et que la littéracie joue un rôle central dans l'acquisition des frontières des mots en présence de resyllabation après 6 ans (Chevrot et al., sous presse ; Ruvoletto, 2016). Les travaux sur l'acquisition des phénomènes de resyllabation en L2 confortent cette idée. Les locuteurs qui apprennent le français comme langue seconde à l'écrit, produisent plus de nonréalisations que les locuteurs L1. De plus, dans le cas d'une réalisation de la liaison, ils choisissent la CL à produire essentiellement sur la base d'une représentation orthographique mémorisée au cas par cas (Wauquier, 2009) ou sont fortement influencés dans sa réalisation à l'oral par la graphie apprise dès les premiers cours de FLE (Harnois-Dalpiano, 2016). De plus, les résultats d'une recherche récente (Wauquier, 2019) montrent que les erreurs en production de liaison commises par des adultes immigrés illettrés, ayant appris le français comme L2 à l'oral uniquement, se rapprochent des erreurs commises par les francophones natifs âgés de deux ans et non pas des erreurs que l'on remarque habituellement chez les apprenants dans les cours de FLE. Ces résultats soulignent la neccessité de prendre en compte la "littéracie" dans le processus d'acquisition des phénomènes de resyllabation.

Sur la base de nos données, nous avons également l'impression que les enfants ne font pas vraiment la différence entre les contextes à $h$ muet et à $h$ aspiré initiaux dans les deux premières années de l'école primaire. Dans notre tâche de perception, nous avons vu que, dans la plupart des cas, les enfants considèrent comme fausse une phrase caractérisée par la non-réalisation de la liaison en présence de $h$ aspiré. Ils corrigent ce qu'ils pensent être une erreur en réalisant la liaison. D'ailleurs, une analyse de Watbled (2013) basée sur les données du corpus PFC que nous avons déjà cité dans l'introduction, montre une augmentation de la fréquence de réalisation de liaisons avec des Mots2 à $h$ aspiré initiale comme dans ils harcèlent [i(l)zaRscl] ou ils hurlent [il.zyRl]. L'auteur explique ces réalisations comme «dues à l'ignorance du statut de mot à $\mathrm{H}$ aspiré » (Watbled, 2013 : 80), mais nous pouvons penser que ces productions sont la conséquence d'une perte de la distinction entre le contexte à $h$ aspiré et le contexte à $h$ muet. D'autres recherches doivent être mises en place pour mieux comprendre le traitement phonologique et la réalisation de la resyllabation dans ces deux contextes, non seulement chez l'enfant, mais également chez l'adulte, en étudiant aussi le rapport entre la production et la compréhension à l'oral et l'orthographe. 
Dans l'introduction, nous avons rappelé l'existence de deux modèles pour décrire l'acquisition des frontières de mots en français : le modèle lexical-constructionniste, qui souligne l'importance de la fréquence des mots dans le lexique pour l'acquisition de leurs frontières (Chevrot et al., 2013), et le modèle morpho-phonologique, qui met en rapport l'acquisition des frontières des mots avec des compétences morpho-phonologiques (Wauquier et Braud, 2005). Les résultats de notre étude ne nous renseignent pas sur lequel des deux modèles serait le meilleur pour expliquer l'acquisition des frontières des mots en français, mais ouvrent la voie à de possibles améliorations et à un débat sur la prise en compte de certaines variables. Comme nous l'avons vu dans l'introduction, ces deux modèles expliquent très bien l'apparition d'erreurs de consonnes erronées chez les jeunes enfants mais n'expliquent pas les erreurs de non-réalisation qui subsistent dans la phase d'acquisition que nous avons étudiée. De plus, ils ne considèrent pas une variable très importante, susceptible d'influencer l'acquisition des frontières des mots, et qui peut expliquer nos résultats : la littéracie. Il est donc envisageable de repenser ces deux modèles pour établir une ou plusieurs étapes intermédiaires permettant de représenter au moyen de structures abstraites ou de gabarits phonologiques les non-réalisations et de prendre en compte l'apprentissage de l'écrit. Prenons par exemple le modèle présenté par Wauquier (2009). Dans ce modèle les enfants encodent la consonne flottante dans la représentation lexicale sous-jacente par bootstrapping morphophonologique. Dans cette explication, le déterminant est acquis vers 5 ans comme un mot qui porte une consonne flottante qui peut être resyllabifiée sur Mot2. Ce processus comporte la disparition des erreurs de liaison en production. Mais alors comment expliquer l'acceptation de nonréalisations dans notre étude? Nous pouvons penser que pour les enfants du CP que nous avons testés, qui commencent l'école et l'apprentissage de l'écrit, les Mot1 et Mot2 sont traités comme deux blocs distincts à l'oral pour la simple raison qu'ils sont séparés par un blanc dans la graphie. Ce traitement pourrait motiver l'acceptation de non-réalisations de resyllabation : comme à l'écrit il y a un blanc, à l'oral il peut y avoir un silence et une juxtaposition de deux mots sans enchaînement. La consonne flottante sera donc encodée mais non réalisée à l'attaque de Mot2, même si ce dernier a une voyelle à l'initiale. Cela implique la nécessité d'introduire dans le modèle de Wauquier (2009) une nouvelle étape pour représenter les non-réalisations, comme l'ont proposé Scheer et al. (2015). Cette nouvelle version du modèle a été enrichie d'une étape 3 (ajoutée à la Figure 2), permettant de représenter les non-réalisations et modélisant une séparation entre l'information segmentale et syllabique où la CL [z] est présente mais non réalisée à l'attaque de Mot2. 


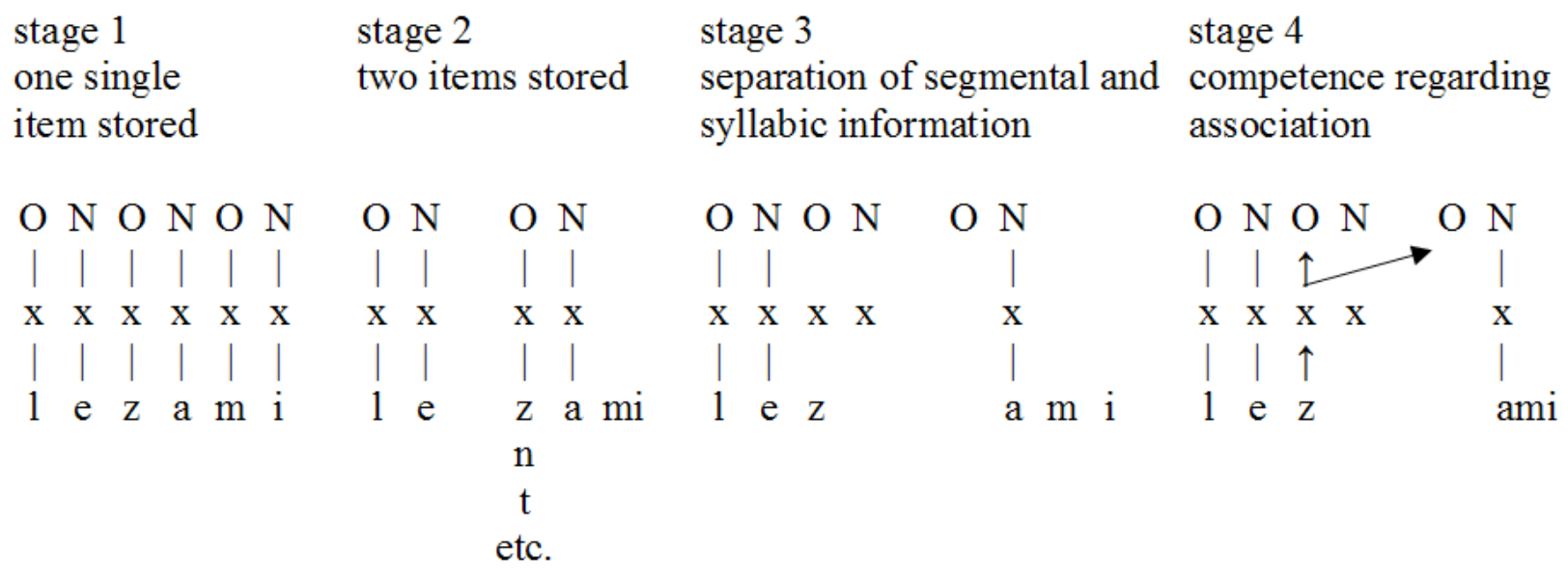

Figure 2. Nouvelle version du modèle phonologique (Scheer, Encrevé et Wausuier, 2015)

La tâche de perception d'erreurs et de reformulation est une tâche métalinguistique qui permet de tester les processus «de type $\mathrm{C}$ », les processus cognitifs où le participant doit être conscient pour traiter l'information (Jack et Shallice, 2001). Étant donné que le processus cognitif qui est testé dans la tâche est un processus de type «conscient», cette tâche ne permet pas d'avoir des informations directes sur la forme sous-jacente. En revanche, elle permet de vérifier des compétences très larges associées à la compréhension et au jugement normatif qui à leur tour sont sujets à des variables individuelles.

Dans l'activité de compréhension, la mémoire à court terme et le niveau d'attention jouent un rôle. Le fait de ne pas avoir détecté une certaine erreur-cible peut être l'effet d'un niveau d'attention trop bas ou d'un problème de mémoire à court terme : la phrase que l'enfant écoute est déjà oubliée en phase de jugement. Par ailleurs, nous avons remarqué que certains enfants n'étaient pas capables de reformuler la phrase écoutée, soit parce qu'ils l'avaient oubliée, soit parce qu'ils ne parviennent pas à se concentrer au moment de l'écoute.

De plus, puisque la tâche teste un processus cognitif conscient et qu'elle a pour but l'identification d'erreurs, les réponses qu'elle implique peuvent être le résultat d'un jugement normatif sur la langue. Un enfant qui commence l'école primaire est déjà familier avec le concept d'erreur, mais ce concept est très loin du concept linguistique de l'«agrammaticalité ». Il est plutôt proche de l'idée de quelque chose d'interdit, d'incorrect ou « qu'il ne faut pas faire ». Les résultats de cette tâche peuvent donc être influencés par le jugement normatif lié à une évaluation plutôt négative de l'erreur, propre aux enfants à cet âge. En plus, comme pour la compréhension, le jugement normatif est à son tour influencé par des variables individuelles comme le contexte social dans lequel l'enfant est inséré, ou encore, l'éducation en famille et à l'école. 
Il faut ajouter qu'une autre limite de notre tâche réside dans le choix du matériel. La têche réunissait trois conditions : non-réalisation, consonne erronée et contrôle. Nous avons vu que, pour l'élision, deux conditions proposent le même type d'erreurs : la armoire, pour la condition de nonréalisation ; le artichaut, pour la condition de consonne erronée. Pour cette dernière condition, une erreur du type le lartichaut [Іə.Іав.ti.fo] aurait a priori pu paraître plus pertinente. Or, le choix de garder l'erreur le artichaut dans la condition de consonne erronée était inspiré par des recherches conduites dans la base de données CHILDES (MacWhinney, 2000). En effet, nous n'y avions repéré aucune occurrence d'erreurs du type le lartichaut.

\section{Références bibliographiques}

BASSET B., 2000, La liaison à 3, 7 et 11 ans : description et acquisition, Thèse de Master, Université Grenoble 3.

Chevrot J.-P., Siccardi A., Parisse C. et Spinelli E., 2014, «Multiword sequences and phonological variants in the lexicon: What can we learn from the acquisition of prenominal liaison in French?», XIX Biennial International Conference on Infant Studies, Berlin, Germany, July 3-5, 2014.

Chevrot J.-P., Chabanal D. et Dugua C., 2007, «Pour un modèle de l'acquisition des liaisons basé sur l'usage : trois études de cas », Journal of French Language Studies, 17, p. 103-128.

Chevrot J.-P., 1996, «Prononciation et situations de lecture : données sociolinguistiques, approches expérimentales, modèles psycholinguistiques », Lidil, 13, p. 127-158.

Chevrot J.-P., Dugua C. et FAyOl M., 2009, «Liaison, word segmentation and construction in French: a usage-based account », Journal of Child Language, 36/3, p. 557-596.

Chevrot J.-P., Dugua C., Harnois-Delpiano M., SicCardi A. et Spinelli E., 2013, «Liaison acquisition: debates, critical issues, future research », Language Sciences, 39, p. 83-94.

CôTÉ M.-H., 2005, «Le statut lexical des consonnes de liaison », Langages, 158, p. 66-78.

CôTÉ M.-H., 2008, «Empty elements in schwa, liaison and h-aspiré: The French Holy Trinity revisited. », in J. M. HARTMANN, HegEdüs V. et VAN RIEMSDIJK H., Sounds of Silence: Empty Elements in Syntax and Phonology, Amsterdam, Elsevier, p. 61-103.

Dugua C. et BAClesse M., 2014, «Incidence d'effets de fréquence sur l'usage de la liaison en lecture à haute voix et dans des jugements normatifs chez des enfants de CE2-CM1 », dans Soum-Favaro C., Coquillon A. et Chevrot J.-P., La liaison : approches contemporaines, Bern, Peter Lang, p. 117-139.

Dugua C., 2006, Liaison, segmentation lexicale et schémas syntaxiques entre 2 et 6 ans. Un modèle développemental basé sur l'usage, Thèse de doctorat, Université Stendhal, Grenoble III. 
Dugua C., Chevrot J.-P, et Fayol M., 2006, «Liaison, segmentation des mots et schémas syntaxiques entre 2 et 6 ans: un scénario développemental », Entretiens de Bichat: Orthophonie, 29-30 septembre 2006, Paris, Expansion, formation et éditions, p. 230-244.

DuRAND J. et LYCHE C., 2008, «French liaison in the light of corpus data », Journal of French Language Studies, 18/1, p. 33-66.

Durand J., Laks B, Calderone B. et Tchobanov A., 2011, «Que savons-nous de la liaison aujourd'hui ? », Langue Française, 169/1, p. 103-126.

ENCREVE P., 1983, «La liaison sans enchaînement », Actes de la recherche en sciences sociales, 46, p. 39-66.

HARNOIS-DALPIANO M., 2016, Le kaléidoscope de la liaison en français : étude comparée de son appropriation par des apprenants adultes de FLE et des enfants natifs, Thèse de doctorat, Université Grenoble Alpes.

JACK A.I. et SHALlice T., 2001, «Introspective physicalism as an approach to the science of consciousness », Cognition, 79, p. 161-196.

LÉtÉ B., Sprenger-Charolles L. et Colé P., 2004, « MANULEX: A grade-level lexical database from French elementary school readers », Behavior Research Methods, 36/1, p. 156-166.

MacWhinney B., 2000, The CHILDES Project: Tools for analyzing talk. Third Edition, Mahwah, NJ, Lawrence Erlbaum Associates.

MALlet G., 2008, La liaison en français : descriptions et analyses dans le corpus PFC, Thèse de doctorat, Université Paris Ouest-Nanterre-La Défense.

NARDY A., 2008, Acquisition des variables sociolinguistiques entre 2 et 6 ans: facteurs sociologiques et influences des interactions au sein du réseau social, Thèse de Doctorat, Université Grenoble 3.

PeEReman R., Dufour S. et Burt J. S., 2009, «Orthographic influences in spoken word recognition: the consistency effect in semantic and gender categorization tasks », Psychon. Bull., 16, p. 363-368.

Ruvoletto S., 2016, Étude longitudinale des phénomènes de resyllabation chez les enfants français entre compétence phonologique, lecture et orthographe, Thèse de doctorat, Université Paris 8 .

SCHANE S.A., 1967, «L'élision et la liaison en français », Adaptation du Chapitre 1 de French Phonology and Morphology, Cambridge, Massachusetts, MIT Press.

SCHEER T., ENCREvÉ P. et WAUQUIER S., 2015, «Autosegmental news from h aspiré and liaison without enchaînement », $13^{\text {th }}$ Conference of the French Phonology Network, Bordeaux, 29 juin - 2 juillet 2015, communication non publiée. Résumé accessible à : http://linguisticsbdx.free.fr/ 
Tomasello M., 2003, Constructing a Language: A Usage-Based Theory of Language Acquisition, Harvard, Harvard University Press.

WATBLED J.-P., 2013, «La liaison en français : dimension structurale et aspects cognitifs de la variation. ? » dans LEDEGEN G., La variation du français dans les espaces créolophones et francophones, France, Europe et Amérique, Paris, L'Harmattan, p. 67-96.

WAUQUiER S., 2009, «Acquisition de la liaison en L1 et L2 : stratégies phonologiques ou lexicales ?», AILE Lia, 2, p. 93-130.

WAUQUIER S., 2010, «Des ornithorynques et consonnes doublement flottantes, pour une théorisation unifiée de la liaison », Faire signe, Volume d’hommages à Pierre Encrevé.

WAUQUIER-GRAVELINES S., 1996, Organisation phonologique et traitement de la parole continue, Thèse de doctorat en linguistique théorique et formelle, UFRL, Paris 7.

WAUQUiER-Gravelines S., 2004, «Les liaisons dangereuses. Psycholinguistique et phonologie : une interface complexe », dans Ferrand, L. et Grainger, J., Psycholinguistique cognitive, Essais en l'honneur de Juan Segui, Bruxelles, De Boeck Université.

WAUquiER-Gravelines S. et Braud V., 2005, « Proto-déterminant et acquisition de la liaison obligatoire en français », Langages, 158, p. 53-65.

WAUQUIER S., 2019, «Liaison 2019 : L'ancien et les nouveaux, quel cumul opérer sur quelles

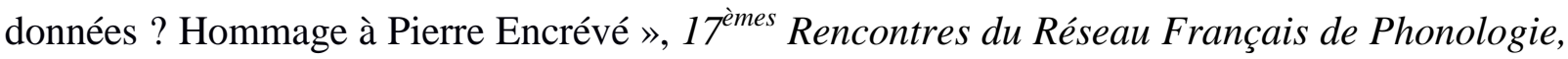
26-28 Juin, Orléans (France), communication non publiée. Résumé disponible sur : https://rfp2019.sciencesconf.org/

\section{ANNEXES}

\section{Annexe 1. Ordre de présentation des phrases dans les blocs}

Comme nous pouvons le voir, nous avons varié l'alternance entre les phrases tests bien formées pour lesquelles nous attendons une réponse affirmative (marquées dans le Tableau 1 par «T, oui »), les phrases tests avec des erreurs pour lesquelles nous attendons une réponse négative (« $\mathrm{T}$, non »), les phrases fillers sans fautes pour lesquelles nous attendons une réponse affirmative ( «F, oui ») et les phrases fillers avec des fautes pour lesquelles nous attendons une réponse négative (« T, non »). 


\begin{tabular}{|c|c|c|c|c|c|c|c|c|}
\hline \multirow[t]{2}{*}{ Blocs } & \multicolumn{8}{|c|}{ Phrases } \\
\hline & 1 & 2 & 3 & 4 & 5 & 6 & 7 & 8 \\
\hline 1 & $\mathrm{~T}$, non & $\mathrm{T}$, non & $\mathrm{F}$, oui & $\mathrm{F}$, non & $\mathrm{T}$, oui & $\mathrm{F}$, non & $\mathrm{F}$, non & $\mathrm{T}$, non \\
\hline 2 & $\mathrm{~T}$, non & $\mathrm{F}$, oui & $\mathrm{T}$, non & $\mathrm{T}$, oui & $\mathrm{F}$, oui & $\mathrm{F}$, non & $\mathrm{F}$, non & $\mathrm{T}$, non \\
\hline 3 & $\mathrm{~F}$, oui & $\mathrm{F}$, non & $\mathrm{T}$, oui & $\mathrm{F}$, non & $\mathrm{T}$, non & $\mathrm{T}$, non & $\mathrm{F}$, non & $\mathrm{T}$, oui \\
\hline 4 & $\mathrm{~F}$, oui & $\mathrm{T}$, oui & $\mathrm{T}$, non & $\mathrm{F}$, oui & $\mathrm{T}$, non & $\mathrm{F}$, oui & $\mathrm{T}$, non & $\mathrm{F}$, non \\
\hline 5 & $\mathrm{~F}$, oui & $\mathrm{T}$, non & $\mathrm{T}$, non & $\mathrm{T}$, oui & $\mathrm{F}$, oui & $\mathrm{F}$, oui & $\mathrm{F}$, oui & $\mathrm{T}$, non \\
\hline 6 & $\mathrm{~T}$, non & $\mathrm{F}$, oui & $\mathrm{T}$, oui & $\mathrm{T}$, non & $\mathrm{F}$, non & $\mathrm{T}$, non & $\mathrm{F}$, oui & $\mathrm{F}$, oui \\
\hline
\end{tabular}

\section{Annexe 2. Phrases fillers}

\begin{tabular}{|c|c|c|c|}
\hline Phrases fillers & Bloc & Réponse & Erreurs et corrections \\
\hline Les petites filles jouent avec des animaux en peluches & 1 & Oui & - \\
\hline J'ai un tapis rouge dans ma chambre & 3 & Oui & - \\
\hline Fabienne et Thibaud sont allés au cinéma hier soir & 6 & Oui & - \\
\hline La marmotte prennent des pissenlits pour se nourrir & 1 & Non & prennent (pour prend) \\
\hline $\begin{array}{l}\text { Moi ce que je préfère quand je vais au parc c'est faire du } \\
\text { togobban }\end{array}$ & 1 & Non & togobban /to.gə.bã/ pour toboggan /to.bə.gã / \\
\hline Les pompiers ont des caks /kaks/ qui brillent & 1 & Non & caks /kaks/ pour casque /kask/ \\
\hline Louis a peur des se(r)pent, surtout les gros & 4 & Non & 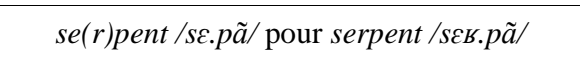 \\
\hline Les enfants aiment les glaces & 5 & Oui & - \\
\hline Les phoques sautent et nagent dans l'eau & 2 & Oui & - \\
\hline Anthony veut manger des biscuits & 4 & Oui & - \\
\hline Michelle et Lucille ont mangé des glaces & 5 & Oui & - \\
\hline Les glaçons est dans le verre & 2 & Non & est pour sont \\
\hline Mon père regarde toujours la télésivion le soir & 6 & Non & 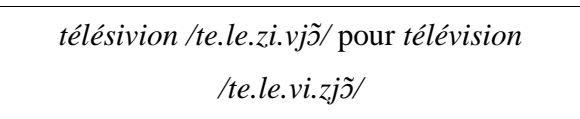 \\
\hline j'ai arrangé mes jouets dans le grand pla[z]card & 2 & Non & pla[z]card /plaz.kas/pour placard/pla.kas/ \\
\hline Les ou(r)s bruns habitent au Canada et ils mangent du miel & 3 & Non & ou(r)s /us/ pour ours /üs/ \\
\hline Maman a cuisiné des pâtes pour nous ce soir & 6 & Oui & - \\
\hline Maman tire les rideaux pour qu'il fasse noire avant de dormir & 2 & Oui & - \\
\hline Le pirate a trouvé un trésor enterré sous le sable & 4 & Oui & - \\
\hline Sylvia et Nadine regardent la télé & 4 & Oui & - \\
\hline Les papillons s'envolent tous ensemble & 5 & Oui & - \\
\hline Mes parents font les courses au mazagin & 3 & Non & 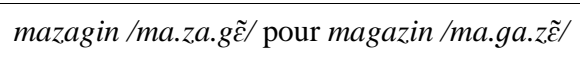 \\
\hline
\end{tabular}




\begin{tabular}{|c|c|c|c|}
\hline La marguerite a des pé[s]tales blanches & 3 & Non & pé[s]tales /pe.stal/ pour pétales /pe.tal/ \\
\hline Moi ce que je préfère à l'école c'est la réc(r)éation & 5 & Non & 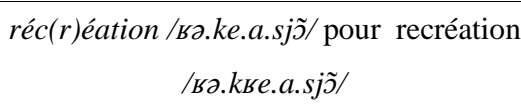 \\
\hline Je vais à l'école en vélo & 6 & Oui & - \\
\hline
\end{tabular}

\section{Annexe 3. Phrases d'entrainement}

\begin{tabular}{|c|c|c|}
\hline Phrases d'entrainement & Réponses attendues & Erreurs \\
\hline Les filles sont très beaux & Non & beaux pour belles \\
\hline Le [n]éléphant est le plus gros animal de la Savane & Non & néléphant/ne.le.fã/ pour éléphant /e.le.fã/ \\
\hline Le chevaux courent dans le bois & Non & le pour les \\
\hline J'ai vu des c(r)ocod[r]iles au zoo & Non & cocodriles /ko.ko.dsil/ pour crocodiles /kьo.ko.dil/ \\
\hline Louis s'est pronemé à la plage & Non & pronemé /рво.пә.me/ pour promené /рво.тә.nе/ \\
\hline Le chien mange ses croquettes & Oui & - \\
\hline Papa m'a a[s]eté un beau vélo & Non & a[s]eté /a.sa.te/ pour acheté /a.fo.tel \\
\hline $\begin{array}{l}\text { Hier au cinéma mes amis ont vu un super dessin } \\
\text { aminé }\end{array}$ & Non & 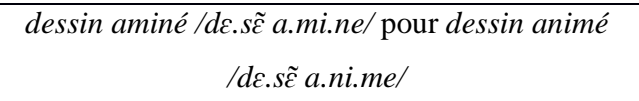 \\
\hline Je mange des biscuits au chocolat & Oui & - \\
\hline Maman aime bien les chats & Oui & - \\
\hline
\end{tabular}

